\title{
LAS IDEAS LINGÜÍSTICAS DE CERVANTES EN EL QUIJOTE •
}

1. El Quijote es también un libro de lingüística. En él se habla muchas veces de lengua, y se exhibe una lengua "histórica" hecha discurso que habla de sí misma. El cervantismo moderno, inaugurado por A. Castro, puso fuera de circulación la idea de que su autor era un «ingenio lego". Cervantes, por el contrario, "se nos muestra plenamente como una de las más espléndidas floraciones del humanismo renacentista" '. También en el campo de las ideas ligüísticas - hay que subrayar-, puesto que el pensamiento lingǘstico constituye un aspecto importante de cualquier cosmovisión. En este sentido interesa a la historia de la lengua recuperar el pensamiento lingüístico de Cervantes. Conocemos bien la lengua del Quijote y su significado, como plasmación de un estilo colectivo, en la evolución histórica del idioma ${ }^{2}$. Pero debemos preguntarnos qué papel representó la teoría lingüística del siglo XVI en la consecución de ese logro histórico que es la lengua del Quijote. Porque las ideas lingüísticas, como componentes del universo cultural y

- Este trabajo fue la comunicación que lei en la VI Conferencia LingüísticoLiteraria de la Universidad de Oriente, de Santiago de Cuba, en febrero de 1989.

1 A. Castro, El pensamiento de Cervantes. Madrid-Barcelona, Ed. Noguer, (Nueva edición ampliada con notas del autor y de J. Rodríguez-Puértolas), 1972, p. 391.

2 Cf. A. Rosenblat, La lengua del "Quijote". Madrid, Gredos, 1978 [1971], 1.• reimpresión, y "La lengua de Cervantes», en J. B. Avalle-Arce y E. C. Riley (eds.), Suma Cervantina. London, Tamesis Books Ltd., 1973, pp. 323-355; R. MENÉNDEZ PIDAL, aEl lenguaje del siglo XVI», en La lengua de Cristóbal Colón, Madrid, EspasaCalpe (Colección «Austral», 280), 1968, 5.• ed., pp. 47-84, y «La lengua castellana en el siglo XVIn, en R. Menéndez Pidal (dir.), Historia de España Madrid, EspasaCalpe, 1986, vol. XXVI, pp. 6-37; H. HATZFELD, El "Quijote" como obra de arte del lenguaje". Madrid, CSIC, 1972, 2." ed. (Anejo 83 de la RFE); L. SPITZER, "Perspectivismo lingüístico en el Quijotex, en Lingüistica e historia literaria Madrid, Gredos, 1968, 2.: ed., pp. 135-187. 
científico de una época, ocupan un primer plano en el contexto histórico-cultural de la evolución de los sistemas lingüísticos.

2. Digamos, en primer lugar, que Cervantes concedió una importancia dẹterminante al tema lingüístico a la hora de componer el Quijote. Éste es un libro de libros ${ }^{3}$. Sus personajes consumen literatura y hablan a menudo de ella. Y al hablar de literatura es forzoso referirse a la lengua literaria. De modo que la presencia del tema de la literatura en el Quijote es el primer argumento para afirmar la importancia del tema lingüístico.

Pero hay más. En la dedicatoria de la II. parte habla el autor de la prisa que le han dado para que la publique; uno de los que más desean leer la continuación del libro

ha sido el gran Emperador de la China, pues en su lengua chinesca habrá un mes que me escribió una carta con un propio, pidiéndome, o, por mejor decir, suplicándome se le enviase, porque queria fundar un colegio donde se leyese la lengua castellana, y quería que el libro que se leyese fuese el de la historia de don Quijote 4.

Entre bromas y veras, Cervantes hace una profecía que se ha cumplido. Si hoy se enseña español en China, es seguro que se enseña en el Quijote. En las palabras recogidas subyace la valoración lingüistica de la propia obra: leer español en China es una forma de afirmar la excelencia de la lengua vernácula, de «nuestro vulgar castellanow, como lo llama otras veces, en la línea de una idea muy común de la lingüística renacentista. Y leerlo en el Quijote es una manera de valorar la lengua de esta obra y el pensamiento lingüístico que la sustenta.

Otro hecho significativo es el prólogo a la I.a parte. El libro ya está escrito, pero Cervantes se plantea cómo escribirlo, insistiendo exclusivamente en la forma lingüística, no en la materia de la narración. Entonces viene el amigo kdiscretow y le soluciona el falso problema con un discurso que, entre otras cosas, contiene una magnifica exposición del ideal de lengua. ¿A qué viene esto, si la primera parte del Quijote ya estaba escrita? No es que el amigo «se pase de listow, como graciosamente comenta Rodríguez Marín. Es que Cervantes, una vez escrito el libro, quiere subrayar la importancia del tema lingüístico. De ese modo queda subrayada la importancia del uso de la lengua en el texto y también la importancia del pensamiento lingüístico que lo sostiene.

Véase A. CASTRO, "La palabra escrita y el "Quijote" ", en Cuadernos de Insula, 1. Homenaje a Cervantes. Madrid, 1947, pp. 9-44.

4 F. Rodríguez Marin (ed.), M. DE CERvantes, El ingenioso Hidalgo Don Quijote de la Mancha, Madrid, Patronato del IV Centenario de Cervantes, 1948, vol. IV, pp. 20-21. Todas las citas del Quijote las tomaré de esta magna edición en diez volúmenes, indicando de ahora en adelante sólo la parte de la novela, el capítulo y la página. 
Y aún podemos traer otro argumento textual para afirmar la importancia del tema lingüístico. El título de muchos capítulos tiene como núcleo palabras que denotan «actos de habla" y los califican muy significativamente: "De la respuesta que dio don Quijote a su reprehensor, con otros graves y graciosos sucesos" (II, 32), «De la sabrosa plática que la Duquesa y sus doncellas pasaron con Sancho Panza, digna de que se lea y de que se note" (II, 33), "Donde se trata del discreto coloquio que Sancho Panza tuvo con su señor don Quijote» (I, 49), «De las discretas altercaciones que don Quijote y el canónigo tuvieron, con otros sucesos" (I, 50), «Del rídiculo razonamiento que pasó entre don Quijote, Sancho Panza y el Bachiller Sansón Carrascow (II, 3), etc. Se me dirá que Cervantes sigue la tradición literaria del diálogo renacentista y que ello es la causa de esos títulos. De acuerdo. Pero eso no niega que Cervantes valorara explícitamente el diálogo como acto de habla. De hecho nada similar encontramos en los títulos de los capítulos de los más importantes diálogos renacentistas que pudieron servirle de modelos: en el Diálogo de Mercurio y Carón de Alfonso de Valdés y en el Diálogo de la lengua de su hermano Juan el título de cada capítulo es la escueta consignación de los nombres de los personajes que dialogan; en el De los nombres de Cristo de Fray Luis de León los capítulos se llaman con el nombre que es objeto de exégesis en cada caso. Es decir, en estas obras los capítulos anuncian a los personajes o resumen el tema, pero no precisan o subrayan el acto de dialogar, como lo hacen en el Quijote sintagmas como "discreto coloquio", "sabrosa plática" 0 . "ridículo razonamiento». Estos sintagmas indican que Cervantes valoraba kel decirn de sus personajes casi tanto o más que «lo dichow. Y esa valoración de la enunciación (y no sólo del enunciado) escondía, sin duda, una preocupación teórica por el ser y funcionar de la lengua.

Intentar exponer cómo el Quijote refleja ciertas ideas lingüísticas de la época y examinar cuál es su posible aportación original al conjunto de esas ideas no es, pues, una pretensión peregrina e infundada 5 . La novela misma nos invita a llevar a cabo esta tarea, pues en su trama argumental y temática la presencia de lo lingüístico queda subrayada en más de una ocasión.

3. Los hechos reseñados demuestran que Cervantes entró de lleno en el problema teórico que la lingüística del Renacimiento italiano denominó questione della lingua. La lingüística española del siglo XVI también se lo planteó ${ }^{6}$. Se trataba de encontrar un

s Para la lingüistica española del siglo XVI, en general, véanse principalmente W. BAHNER, La lingüistica española del Siglo de Oro, Madrid, Ciencia Nueva, 1966, y R. HAmilton, "Juan de Valdés and some Renaissance Theories of Language*, Bulletin of Hispanic Studies, XXX, 1953, pp. 125-134.

- Véase E. L. Rivers, «Cervantes and the question of Languagew, en.M. D. McGaha (ed.), Cervantes and the Renaissance. Papers of the Pomona College Cervantes Symposium. Easton, Pennsylvania, Juan de la Cuesta, 1980, pp. 23-33. 
ideal de lengua, una norma culta que garantizara la realización del sistema en las condiciones de dignificación del vulgar apropiadas para que éste fuera del todo equiparable al latín. La empresa no fue otra cosa que una magna revolución cultural y lingüística, pues había que romper el estado de diglosia medieval del latín y el romance. Y había que romperlo a favor de éste último, la lengua de menor prestigio socio-cultural. En esta empresa participan gramáticos y escritores, porque la kcuestión de la lengua" en España - a diferencia de Italia - se convierte en una "cuestión de la literatura", como ha señalado L. Terracini ${ }^{7}$. Boscán para la prosa - con la traducción del Cortesano ${ }^{8}$ - y Garcilaso para la poesía crean el ideal de lengua renacentista, cimentado en el habla de Toledo. Valdés, en su Diálogo, eleva ese ideal a rango de teoría. Cervantes conoció la obra de Garcilaso y la de Boscán, aunque - como apunta E. L. Rivers ${ }^{9}$ - no es probable que conociera el Diálogo de la lengua. En cualquier caso, la aportación cervantina es sumamente original en el Quijote. Aquí se presenta un ideal de lengua fundado en los principios de naturalidad y selección:

El lenguaje puro, el propio, el elegante y claro está en los discretos cortesanos, aunque hayan nacido en Majadahonda; dije discretos porque hay muchos que no lo son, y la discreción es la gramática del buen lenguaje, que se acompaña con el uso (II, 19, 95-96).

Ese ideal de lengua conlleva el rechazo del latín y del latinismo, el empleo de refranes como manifestación de la naturalidad y espontaneidad y, sobre todo, el uso de una lengua internamente muy diferenciada, en la que tienen cabida todas las variedades, desde el vulgarismo al cultismo. Y esta es la gran aportación de Cervantes en el Quijote a la cuestión de la lengua. De modo que en esa novela está el mayor comentario acerca del lenguaje del Renacimiento y post-Renacimiento. Don Quijote muestra el ideal renacentista; Sancho, el medieval; y el autor del prólogo a la I." parte, el barroco. Así, en el campo de la teoría - pero una teoría no expuesta didácticamente, sino convertida en vida de ficción-, Cervantes significa un avance con respecto a la doctrina lingüística de su época, codificada en el Diálogo de Valdés.

4. Veamos cómo en el Quijote se exhibe, se cita, la interna diferenciación del idioma. Cervantes, en consonancia con algunos lingüistas de su época, intuye un asunto que interesa hoy a la

1 Véase «Tradizione illustre e lingua letteraria, problema del Rinascimento spagnolow, en Lingua come problema nella letteratura spagnola del Cinquecento, Torino, Stampatori, 1979. Y también C. Barbolani (ed.), JUAN DE VALDÉs, Diálogo de la lengua, Madrid, Cátedra, 1982, p. 92.

- Véase M. Morreale, Castiglione y Boscán: el ideal cortesano en el Renacimiento español Madrid, Anejos del BRAE, 1959, 2 vols.

- "Cervantes and the Question of Languagew, p. 25. 
moderna historia de la lengua, a la dialectología y a la sociolingüística. A saber: las lenguas funcionan por medio de variedades internas; el hablar nunca es "purow, en sentido estrico, porque la lengua "real" o «histórica" es un diasistema de variedades diatópicas, diastráticas y diafásicas, es, por tanto, un código esencialmente heterogéneo. Dentro de esa heterogeneidad tienen cabida incluso las formas más plebeyas, según la idea neoplatónica que pregona la belleza de la naturaleza creada en todas sus variopintas formas. M. K. Read ${ }^{10}$ ha señalado la resonancia de esta idea en el ámbito de la lingüística española del Renacimiento, y ha puesto como botón de muestra al maestro Correas:

\begin{abstract}
Ha-se de advertir qe una Lengua tiene algunas diferenzias, fuera de dialectos particulares de provinzias, conforme á las edades, calidades, i estados de sus naturales, de rústicos, de vulgo, de ziudad, de la jente más granada, i de la Corte, del Historiador, del anziano, i Predicador, i aun de la menor edad, de mujeres i varones: i todas estas abraza la Lengua universal debajo de su propiedad, niervo i frase; i á cada uno le está bien su lengua, $i$ al Cortesano no le está mal escojer lo ge le pareze mejor á su propósito, como en el traje. Mas no por eso se ha de entender qe su estilo particular es toda la lengua entera $i$ jeneral, sino una parte; porque muchas cosas qe él desecha, son mui buenas i elegantes para el historiador i anziano i Predicador i los otros.
\end{abstract}

Pero antes de que el maestro de Salamanca consigne en su Arte estas observaciones, ya Cervantes en el Quijote muestra narrativamente cómo el castellano vive en contacto con otras lenguas y es, al mismo tiempo, un diasistema internamente muy diferenciado.

Veamos, primero, los contactos lingüísticos.

El castellano vive en contacto con el latín. Primero, porque el narrador y los personajes, mezclan a veces su español con palabras y frases latinas; segundo, porque en otras ocasiones toman palabras directamente del latín y las españolizan, es decir, usan cultismos. La primera modalidad -el empleo de latines en el discurso- está sutilmente condenada, porque se ridiculiza o se toma con ironía. Así, los latines de don Quijote, de Sansón Carrasco, del médico de Barataria Pedro Recio o de Maese Pedro. Hay que añadir que lo que se ridiculiza es el propio latín y, especialmente, el latín eclesiástico. Por ejemplo, en el baile que se da en casa de don Antonio Montero don Quijote rechaza las insinuaciones de algunas damas ligeras y alegres con un "iFugite, partes adversae!" (II, 62, 76), tomado de los exorcismos eclesiásticos. Y el bribón de Maese Pedro acude nada menos que al Evangelio de San Juan (X, 38), al decir «y operibus credite, et non verbis, y manos a la labor" (II, 25, 231). Lo mismo podría decirse de otros muchos casos, incluso

10 "The concept of Man as "Homo loquens" in the Spanish Renaissance", RSEL, 12 1982, pp. 65-84. La cita de CoRREAS - Arte grande, ed. de E. Alarcos García, pp. $60-61$ - en p. 82. 
cuando el latín empleado no es el eclesiástico, sino el escolástico. En esta condena subyace, también, la defensa de la lengua vulgar: todo lo que se dice en latín se puede decir en castellano.

Otra cosa es el cultismo. Sancho no entiende la palabra longincuos que acaba de empler: : su señor. Y don Quijote explica: «Longincuos quiere decir apartados, y no es maravilla que no lo entiendas; que no estás tú obligado a saber latín, como algunos que presumen que lo saben, y lo ignoran" (II, 29, 294). El cultismo ejemplifica un contacto de lenguas típico: una palabra se toma del latín y se introduce en el español, adaptándola morfológicamente. El cultismo, además, distingue socialmente; es una variedad diastrática de las clases socioculturalmente altas; por eso no es maravilla que Sancho no lo entienda. Ahora bien, con el tiempo y el uso el cultimo puede dejar de ser variedad diastrática y convertirse en modalidad común (en lengua estándar, diríamos hoy): es preferible erutar a regoldar, dice don Quijote: «la gente curiosa se ha acogido al latín, y al regoldar dice erutar, y a los regüeldos, erutaciones, y cuando algunos no entienden estos términos, importa poco; que el uso los irá introduciendo con el tiempo, que con facilidad se entiendan; y esto es enriquecer la lengua, sobre quien tiene poder el vulgo y el uson (II, 43, 250-51).

El español entra también en contacto con el árabe dentro del ámbito novelístico del Quijote. El texto se hace eco de ese contacto de varios modos.

En primer lugar, el narrador indica - por medio de expresiones como dijo en arábigo, en lengua morisca, en su lengua- que un determinado personaje habla en árabe, ya sea en discurso directo o indirecto; pero las palabras referidas son lógicamente españolas. En estos casos, el árabe no está mencionado o mostrado, sino sugerido; esas indicaciones sirven para que el lector se imagine una caracterización lingüística del personaje. pero al mismo tiempo hacen patente el interés de Cervantes por el contacto de lenguas como tema de su novela. Esto se ve muy bien cuando el narrador, innecesariamente desde el punto de vista del argumento, acumula detalles para dar una imagen exacta de la lingua franca cristianoárabe:

...el cual me dijo en lengua que en toda la Berberia, y aun en Constantinopla, se habla entre cautivos y moros, que ni es morisca, ni castellana, ni de otra nación alguna, sino una mezcla de todas las lenguas, con la cual todos nos entendemos, digo, pues, que en esta manera de lenguaje me preguntó que qué buscaba en aquel su jardín, y de quién era $(1,41$, 210).

0 cuando describe el bilingüismo perfecto del morisco Ricote: "y Ricote, sin tropezar nada en su lengua morisca, en la pura castellana le dijo las siguientes razones:-Bien sabes..." (II, 54, 215).

Un segundo modo de indicar el contacto del árabe con el español consiste en poner alguna palabra o frase árabes en el 
discurso directó o indirecto de un personaje. De este modo queda caracterizado lingüísticamente de una manera más viva e intensa que antes. Los ejemplos son muy abundantes en discurso directo; Cervantes sigue una tradición, documentada ya en los textos medievales: "Gualá ('por Alá), cristiano, que debe ser muy hermosa..." (I, 41, 215), en boca del padre de Zoraida; " ¿Tamxixi cristiano? ¿Tamxixt? -Que quiere decir: ' ¿Vaste, cristiano...?'” (Id.: 216), en boca de Zoraida. Incluso emplea las mismas palabras árabes que en el siglo XIV el Arcipreste de Hita puso en labios de su mora: "Amexi cristiano; amexi 'Vete, cristiano, vete'" (Id.: 217). Más original se muestra Cervantes cuando utiliza la misma caracterización en el discurso indirecto: «Zoraida... preguntó con voz baja si éramos nazarani como si dijera o preguntara si éramos cristianos" (Id.: 220).

Hay un tercer modo. Consiste en mostrar indirectamente la presencia del árabe en contacto con el español por medio de un discurso español que denota el escaso conocimiento de esta lengua por parte del hablante; dice Zoraida: «iNo, no Zoraida: María, Maria!", y comenta el narrador: "dando a entender que se llamaba María, y no Zoraida» (I, 37, 148).

Por último, un cuarto modo, más importante lingüísticamente, es el empleo por el narrador o por un personaje de una palabra o frase árabes, a la que puede o no seguir la traducción o glosa: "zalá cristianesca" ('oración'); "jumá, que es el viernes", etc. Los ejemplos son muy numerosos. A veces el narrador se complace en mostrar la dialectología árabe, acumulando variedades geográficas que designan una misma realidad: "Tagarinos llaman en Berbería a los moros de Aragón, y a los de Granada, mudéjares; y en el reino de Fez llaman a los mudéjares elches (I, 41, 208). Don Quijote hace lexicografía cuando define la palabra albogues y otros arabismos léxicos del español, aunque se equivoca, pues no todas las palabras que comienza por al-son de origen árabe, como ya había señalado Juan de Valdés. Pero que se equivoque don Quijote en asuntos de lengua es significativo. El ideal de lengua de Cervantes es también, como otros temas de la novela, producto de una integración de perspectivas, y no puede encarnarse en un solo personaje, aunque éste sea el protagonista.

El español, en el Quijote, entra en contacto, así mismo, con el italiano. Don Quijote conoce el italiano y se jacta de ello en la imprenta de Barcelona. Emplea italianismos en su español ("iqué peje pillamo?", II, 25, 224), como el Ventero de la II." parte usa galante y bon compaño (II, 25, 223). El italiano sirve también a los alemanes para comunicarse con los españoles; pero lo curioso es que el español de Sancho sufre, en su morfología, ese contacto con el italiano de los germanos, así como ese italiano está en parte españolizado. Dicen los que van con Ricote: «Españoli y tudesqui, tuto uno: bon compaño, y Sancho respondía: - iBon compaño, jura Dih (II, 54, 214). En el enunciado de los alemanes. aparece la conjunción española $y$, en el de Sancho, la forma $D i$ por Dios. 
De un modo mucho más indirecto y con menor relevancia aparecen en el Quijote otras lenguas en contacto con el español: el alemán, el francés, los idiomas peninsulares. De estos últimos, el vascuence o eusquera contamina al castellano del vizcaíno, con unos rasgos que fueron tópicos en la literatura de la época y que al parecer ni siquiera se ajustan a la realidad; el lenguaje aragonés se menciona una vez, lo mismo que el catalán, pero sin que se reproduzca palabra ".

Véamos ahora las variedades internas del español.

Dentro de la modalidad lingüística fundamental -que es el castellano culto de la época, el castellano de los "discretos cortesanosw, aunque hayan nacido en Majadahonda-, en la lengua del Quijote coexisten importantes variedades internas: hay vulgarismos en las hablas de Sancho ${ }^{12}$ y su mujer; hay hablas jergales (de germanía, de estudiantes y pícaros, etc.) en el discurso de los personajes y en el del narrador; hay intertextualización de discursos sociales (expresiones populares, modismos, tecnicismos jurídicos consolidados en el habla popular $y$, por supuesto, refranes) en las elocuciones de personajes y de narrador; y hay, en fin, intertextualización de determinados discursos literarios (arcaísmos de las novelas de caballerías, naturalidad expresiva del Romancero, rasgos de la lengua literaria de Garcilaso, de la novela pastoril, etc.). No podemos entrar en el análisis detallado de toda esta diferenciación interna del castellano de Cervantes. Pero señalemos algunos puntos de interés.

¿A qué se debe este gusto por reflejar la lengua real? En primer lugar, a la caracterización lingüística del personaje, una técnica que ya se empleaba en el teatro de la época. Pero Cervantes la desarrolla y la convierte en tema central de su novela. Porque en el teatro es el gracioso, por ejemplo, el que trabuca las palabras y el que usa vulgarismos léxicos y morfológicos. Pero en el Quijote la diferenciación interna del idioma alcanza a todos los personajes y al narrador. El arcaísmo caballeresco de don Quijote (malferido, fuyades, etc.) está también en boca de su sobrina, de Sancho, del narrador. Hay un cuidado muy notable por señalar todas esas variedades internas: "éste [andar] que entre ellos llaman andar a la sopan (I, 37, 153); «la flor de las ceremonias, o cirimonias, como vos decísu, dice la Duquesa a Sancho (II, 32, 66), «No se le cocía el pan a don Quijote, como suele decirse..." (II, 25, 209), etc. No es simple gusto por exhibir la lengua como un diasistema de varieda-

"Tomo estos datos de C. Fernández Gómez, Vocabulario de Cervantes. Madrid, RAE, 1962: «La otra, que el lenguaje es Aragonésw, Quij., II, III, 227 (s. v. lenguaje); "Diziéndoles en lengua Catalana que estuiessen quedos", Quij., II, III, 230 (s. v. lengua). Es curioso: según los ejemplos de Fernández Gómez, andaluz, gallego y leonés se utilizan sólo como gentilicios en el Quijote, navarro no aparece, ni como gentilicio ni como nombre de un lenguaje o dialecto.

12 Véase A. Alonso, «Las prevaricaciones idiomáticas de Sancho», Nueva Revista de Filología Hispánica, II, 1948, pp. 1-20. 
des internas; por medio de esta lengua, diferenciada y varia, se expresan puntos de vista diferentes que integran el perspectivismo esencial de la obra. La palabra baciyelmo - un compuesto morfológica y léxicamente aceptable, pero con una difícil referencia en el "mundo real" de la narración- es un buen símbolo de esta integración de perspectivas sociolingüísticas, como ha señalado recientemente $\mathbf{E}$. L. Rivers ${ }^{13}$. El narrador pone en distanciamiento irónico la veracidad de su propia narración por medio de las variedades lingüísticas internas: en el capítulo 5 de la II.a parte sugiere, más de una vez, la posibilidad de que sea apócrifo, porque en él Sancho habla mejor de lo que le corresponde. He aquí una prueba palpable de cómo la lengua castellana funciona en el texto como tema de la novela.

Pero la caracterización lingüística no agota el tema lingüístico. El narrador muestra con insistencia un hecho que debe tener su importancia argumental y temática: a menudo los personajes no entienden el habla de los otros, o no son entendidos por los demás. No es entendido el lenguaje caballeresco de don Quijote, lenguaje que se califica, por parte de sus interlocutores, de disparate, de jerigonza, de griego, de retóricas, etc. Tampoco Teresa Panza o el morisco Ricote entienden el lenguaje caballeresco del Sancho quijotizado; ni la Duquesa entiende el culto y neológico sintagma "retórica demostina" (II, 32, 48-49), que emplea don Quijote; por otra parte, este mismo sintagma es de formación discutible, al menos, pues cabría esperar "demostenesiana", de Demóstenes, lo mismo que de Descartes (o de Cartesius) se forma cartesiano. Por otra parte, el mismo don Quijote no entiende el lenguaje de germanía ("gurapas", por galeras, "cantar", por delatar); ni siquiera entiende ciertos registros de la lengua común -como el eufemismo "hacer aguas", con el que "en la escuela destetan a los muchachos", como lo hace ver Sancho (I, 48, 367)-, o ciertas variedades locales generalizadas también en la lengua de todos, como "truchuela" (para designar el 'abadejo', y no una 'trucha'). Este no entender y no ser entendido está señalando la variedad interna de la lengua y, al mismo tiempo, que el ideal de lengua no es patrimonio de ningún individuo, ni siquiera de ninguna clase social.

Finalmente, la variedad interna del idioma se exhibe y se cita en el Quijote todavía de otro modo indirecto. Con muchísima frecuencia los personajes corrigen a los otros personajes la elocución o la construcción del discurso. Don Quijote corrige los vulgarismos de Sancho y de los cabreros, o el tono de sus discursos, o su prolijidad innecesaria; Sansón Carrasco corrige también a San: cho; el Barbero corrige al Ventero; y el mismo Sancho corrige a su mujer, o al Labrador de Barataria, o, incluso, la imprecisión del bachiller Sansón Carrasco. Estas correcciones son un remedo de

13 «El gran acierto del Quijote», Insula, 488-89, 1987, pp. 1 у 4. 
la afición a reprochar el mal uso lingüístico, muy frecuente en el teatro y, sin duda, en la vida cotidiana como apunta A. Rosenblat ${ }^{14}$. Pero también ejemplifican el valor social de la lengua, un valor de gran importancia en la época, como se pone de manifiesto en otras obras: citemos, por poner un solo ejemplo, el Galateo español, de Lucas Gracián Dantisco. Y es que, como obsevan A. Alonso y A. Rosenblat, "hablar bien era entonces un valor nuevo, que formaba parte de la buena crianza, un valor social e individual que el siglo XVI había convertido en primordial" 15.

Pero las correcciones suscitan la réplica de los corregidos. La sociolingüística actual ha acuñado la noción y el término de actitud lingüisitica para denotar la toma de posición del hablante ante su propia lengua, una toma de posición que puede ser positiva o negativa ${ }^{16}$. Es curioso que en el Quijote, la mayoría de las veces, el personaje corregido adopta una actitud lingüística prositiva, de defensa de su propia habla y de identificación intelectual y afectiva con la misma. El cabrero, Sancho, la mujere de Sancho defienden su hablar incorrecto o vulgar ante quien les reprocha, y se enfadan (actitud emotiva) por lo que consideran "niñerías" del reprochador (actitud intelectual sui generis). Una vez Sancho muestra una actitud negativa ante su lengua: cuando promete a don Quijote dejar de decir regoldar y acogerse al más culto erutar. Aun así, le traiciona en seguida su conciencia sociolingüística y cae de nuevo en la palabra estigmatizada. Pero es que aquí hay más de un asunto: por una parte, la condena de regoldar es más asunto del idiolecto de don Quijote que de la valoración social de la época, pues ni Covarrubias ni Gracián Dantisco, por ejemplo, muestran la aversión de don Quijote por el vocablo; por otro lado, Sancho está en vísperas de acceder a su Gobierno de la Insula, esto es, de subir en la escala social. Y, claro, acepta cualquier corrección lingüistica que lo pueda mejorar, incluso a costa de renunciar a su propia conciencia sociolingüística.

La exhibición de estas actitudes lingüísticas en los personajes significa que Cervantes valora, por sí misma, la lengua real y sus diferencias internas. Frente a la condena medieval de los dialectalismos geográficos y sociales -encarnada en la máxima autoridad de Dante-, el renacentista Cervantes - como señaló L. Spitzer«los considera modos de expresión que existen como realidades individuales y que en sí mismos tienen su justificación" ${ }^{27}$. Y, por esto mismo, la lengua real de la época se convierte en tema esencial de su novela.

14 La lengua del "Quijote". Madrid, Gredos, 1978, p. 34.

is Idem: ibidem.

16 H. LOPEZ MORALES, «Sociolingüística Hispánica: perspectivas futuras», en J. M. Lope Blanch (ed.), Perspectivas de la investigación lingüistica en Hispanoamérica. Memoria México, UNAM, 1980, 59-78, pp. 62-64.

17 "Perspectivismo lingüistico en el Quijoten, p. 160. 
5. Debo concluir. No he hecho sino repasar algunos de los temas lingüísticos del Quijote. Ni siquiera todos. Por ejemplo, la traducción es un asunto por el que se interesaron los lingüistas de la época. Y en la novela aparece también una doctrina de la traducción ${ }^{18}$. Pero de momento señalemos tres conclusiones: 1) Cervantes no se limita a reproducir un pensamiento de época, sino que lo elabora originalmente; por eso es un autor que interesa a la historia de las ideas lingüísticas y a la historia de la lengua; 2) el logro teórico más importante de Cervantes es la inclusión en el ideal de lengua de la lengua histórica como diasistema de variedades internas, en lo cual se adelanta al maestro Correas; 3) la teoría lingüística está en la base de la creación de la novela moderna; el tema de la lengua, en su complejidad, es un tema central de la novela moderna. 